\title{
Characterization of oral bacterial diversity of irradiated patients by high-throughput sequencing
}

\author{
Yue-Jian $\mathrm{Hu}^{1,2}$, Qian Wang ${ }^{1}$, Yun-Tao Jiang ${ }^{1}$, Rui Ma ${ }^{1}$, Wen-Wei Xia ${ }^{1}$, Zi-Sheng Tang ${ }^{1}$, Zheng Liu ${ }^{1}$, \\ Jing-Ping Liang ${ }^{1}$ and Zheng-Wei Huang ${ }^{1}$
}

\begin{abstract}
The objective of this study was to investigate the compositional profiles and microbial shifts of oral microbiota during head-and-neck radiotherapy. Bioinformatic analysis based on 16S rRNA gene pyrosequencing was performed to assess the diversity and variation of oral microbiota of irradiated patients. Eight patients with head and neck cancers were involved in this study. For each patient, supragingival plaque samples were collected at seven time points before and during radiotherapy. A total of 147232 qualified sequences were obtained through pyrosequencing and bioinformatic analysis, representing 3460 species level operational taxonomic units (OTUs) and 140 genus level taxa. Temporal variations were observed across different time points and supported by cluster analysis based on weighted UniFrac metrics. Moreover, the low evenness of oral microbial communities in relative abundance was revealed by Lorenz curves. This study contributed to a better understanding of the detailed characterization of oral bacterial diversity of irradiated patients.
\end{abstract}

International Journal of Oral Science (2013) 5, 21-25; doi:10.1038/ijos.2013.15; published online 29 March 2013

Keywords: dental plaque; head-and-neck radiotherapy; microbial diversity; oral microbiota; pyrosequencing

\section{INTRODUCTION}

Radiation therapy remains the primary treatment modality used for patients with head and neck cancer. Many patients are submitted to high doses of radiotherapy of large areas including dentition, oral mucosa, maxilla, mandible and salivary glands. ${ }^{1-2}$ As a result of direct or indirect effects of ionizing radiation, oral complications such as radiation caries, mucositis, candidiasis and soft tissue necrosis are inevitable, which in turn lead to a decrease in quality of life. Previous studies on the mechanisms of radiation-induced oral complications mainly focused on radiation dosimetry, effects on DNA, changes in salivary flow and quality, etc. ${ }^{3-5}$ In addition, perturbation on the microbiota may also contribute to an imbalance in the oral microecosystem and play an important role in oral health maintenance. ${ }^{6}$ Thus, a better understanding of the oral microbiota is essential for effective preventive oral care programs in relation to patients receiving radiotherapy.

Dental plaque harbors a highly diverse resident community of microorganisms. A few decades ago, most of our knowledge on the composition of the oral microbiota was based mainly on culture techniques. ${ }^{7}$ With the advent of molecular techniques such as denaturing gradient gel electrophoresis (DGGE) and terminal restriction fragment length polymorphism (T-RFLP), it has been found that culture-dependent methods appear to underestimate oral microbiota diversity. To date, more than 700 taxa have been identified from the oral microbiota based on information derived from culture and molecular approaches. ${ }^{8-9}$ Culture-independent molecular techniques are capable of surveying entire bacterial communities and characterizing an enormous diversity of oral microbiota, bypassing the need to culture bacteria. To avoid inefficiency and underestimation caused by cultivation techniques, molecular fingerprinting and high-throughput sequencing have already been used to explore changes of oral microflora in healthy individuals, ${ }^{10-12}$ but have not so far been applied to assess radiation-induced shifts of the oral ecosystem.

In this study, a high-throughput sequencing technique-pyrosequencing — was used to estimate the detailed diversity of plaque microbiota of irradiated patients. Moreover, this study aimed to assess the temporal variation in plaque microbiota during radiotherapy.

\section{MATERIALS AND METHODS}

Subjects characteristics and radiotherapy protocols

This study was approved by the ethics committee of Shanghai Jiao Tong University. After we had obtained written informed consent, eight patients who were scheduled to receive head-and-neck radiation therapy were included as study subjects at our institution. The average age was about 45 years, with life expectancy of at least 2 months. Patients who received previous head-and-neck irradiation or chemotherapy were excluded. None of the patients received antibiotics during therapy or within 3 months before the study, nor did they have Sjögren's syndrome ${ }^{13}$ or any disease characterized by xerostomia. The protocol of radiation technique has been previously described in detail

\footnotetext{
${ }_{1}^{1}$ Department of Endodontics, Ninth People's Hospital, Shanghai Jiao Tong University School of Medicine, Shanghai Key Laboratory of Stomatology, Shanghai, China and ${ }^{2}$ Department of Endodontics, The Affiliated Stomatology Hospital of Tongji University, Tongji University School of Stomatology, Shanghai, China

Correspondence: Dr ZW Huang; Dr WW Xia, Department of Endodontics, Ninth People's Hospital, Shanghai Jiao Tong University School of Medicine, Shanghai Key Laboratory of Stomatology, No. 639 Zhizaoju Road, Shanghai 200011, China.

E-mail: huangzhengwei@shsmu.edu.cn; xwwall@sina.com

Received 26 August 2012; accepted 14 January 2013
} 
Table 1 Comparison of phylotype coverage and diversity estimation at 3\% dissimilarity in observed diversity richness (OTUs), estimated OTU richness (ACE and Chao 1), Good's coverage (Good) and diversity indices (Shannon and Simpson)

\begin{tabular}{|c|c|c|c|c|c|c|c|}
\hline Time point & Average number of sequences per sample (s.d.) & OTUs & ACE & Chaol & Good & Shannon & Simpson \\
\hline PT & $2823(138.8)$ & 1024 & 2344 & 1866 & 0.979 & 4.46 & 0.0384 \\
\hline 10 Gy & $3031(148.1)$ & 1038 & 2420 & 1882 & 0.980 & 4.61 & 0.0291 \\
\hline 20 Gy & $2251(180.2)$ & 836 & 2190 & 1610 & 0.976 & 4.20 & 0.0402 \\
\hline 30 Gy & $2618(144.7)$ & 895 & 2016 & 1544 & 0.981 & 4.51 & 0.0294 \\
\hline 40 Gy & 2983 (183.7) & 731 & 1780 & 1365 & 0.985 & 3.87 & 0.0504 \\
\hline 50 Gy & 2736 (143.3) & 815 & 2244 & 1633 & 0.981 & 4.00 & 0.0474 \\
\hline 60 Gy & 1963 (101.4) & 580 & 1515 & 1103 & 0.982 & 3.96 & 0.0410 \\
\hline
\end{tabular}

by Shao et al. ${ }^{14}$ The primary field was irradiated through lateral parallel-opposed portals with 6-MV photons, 2.0 Gy/30 fractions. Each patient received 10 Gy per week for 6 weeks, with cumulative dose of $60 \mathrm{~Gy}$. The parotid and submandibular glands were directly adjacent to the target volume and could not be spared.

\section{Microbial sampling}

For each of the eight subjects, supragingival plaque samples were collected at seven time points (once per week for 7 weeks) before and during radiotherapy using the method mentioned in the Manual of Procedures for Human Microbiome Project (http://hmpdacc. org/tools_protocols/tools_protocols.php) with minor modifications. Plaque samples were obtained from the maxillary first molar according to the above mentioned protocols. Briefly, after the sampling site had been isolated with cotton rolls and dried with a gentle stream of air from an air-water syringe, a sterile Gracey curette was used to remove all of the supragingival plaque from the buccogingival surfaces of the maxillary first molar with as many strokes as necessary. The collected plaque sample was released from the curette by agitation in $300 \mu \mathrm{L}$ of TE buffer $\left(10 \mathrm{mmol} \cdot \mathrm{L}^{-1}\right.$ Tris- $\mathrm{HCl}(\mathrm{pH} 7.5)$ and $1 \mathrm{mmol} \cdot \mathrm{L}^{-1}$ ethylene diaminetetraacetic acid). The microbial samples were immediately transported on ice to the laboratory for further DNA extraction and pyrosequencing analysis. All samples were collected at seven time points within 7 weeks. The samples collected at the time point PT (prior to treatment, no dose received) was used as a control group. The following 6-week treatment period included 10 Gy (the first week of radiotherapy), $20 \mathrm{~Gy}$ (second week), $30 \mathrm{~Gy}$ (third week), $40 \mathrm{~Gy}$ (fourth week), 50 Gy (fifth week) and 60 Gy (sixth week, the end of radiotherapy).

\section{DNA extraction and pyrosequencing analysis}

The plaque samples were lysed in a Mini-Beadbeater-16 (Biospec Products, Bartlesville, OK, USA) according to the manufacturer's instructions. The total genomic DNA was obtained from the lysate using a Bacterial Genomic DNA Extraction Kit (QIAGEN, Valencia, CA, USA). All DNA was stored at $-20{ }^{\circ} \mathrm{C}$ before further analysis. Polymerase chain reaction (PCR) amplification of the $16 \mathrm{~S}$ rDNA hypervariable $\mathrm{V} 1-\mathrm{V} 3$ region $^{12}$ was carried out using the forward primer $8 \mathrm{~F}$ and reverse primer $533 \mathrm{R}$, and pyrosequencing was performed with standard Roche 454 GS-FLX protocols. ${ }^{15}$ The primer sequences and 8-bp barcode were removed. The sequences that were less than $200 \mathrm{bp}$, contained ambiguous bases or homopolymeric stretches, or checked as chimeric artifacts were discarded. The qualified sequences were submitted to the SILVA database (SILVA 106; http://www.arbsilva.de) for taxonomic analysis. MOTHUR (version 1.25.1; http:// www.mothur.org/) was applied to generate the operational taxonomic units (OTUs) and OTU rarefaction curves. Community richness and diversity indices (ACE, Chao1, Good's coverage, Shannon Weaver and Simpson diversity indices) were also determined by the MOTHUR program at the 0.03 level. The profile heat map was generated by the
R program (http://www.r-project.org/). Lorenz curves were created in Excel (Office 2007; Microsoft Corporation, Redmond, WA, USA).

\section{RESULTS AND DISCUSSION}

Overall sequence data

A total of 189305 sequences were generated and 147232 qualified sequences were used for further analyses. A total of 3460 OTUs were identified from all samples based on $3 \%$ sequence dissimilarity. The average number of sequences at each time point was 21033 (2 629 per sample, s.d. $=392.9$ ). Good's coverage was around $98 \%$ for the all sequences at seven time points, indicating that about two additional phylotypes would be expected for every 100 additional sequences obtained (Table 1). This level of coverage indicated that the $16 \mathrm{~S}$ rRNA sequences identified at these time points represented most of the bacterial sequences present in the plaque samples. ${ }^{16}$ The richness of bacterial communities of plaque before and during radiotherapy was estimated by rarefaction curves. For instance, the rarefaction curves of control group (PT, Figure 1) presented different slope at three dissimilarity levels $(3 \%, 5 \%$ and $10 \%)$, which reveals the relationship between OTUs and sampling depth. Generally, the cutoff of $3 \%$ dissimilarity was used in species level analyses. However, the steep slope on the rarefaction curve at 3\% cutoff suggested that the number of OTUs or the bacterial richness of the plaque samples was not yet completely revealed by the current number of sequences. How many

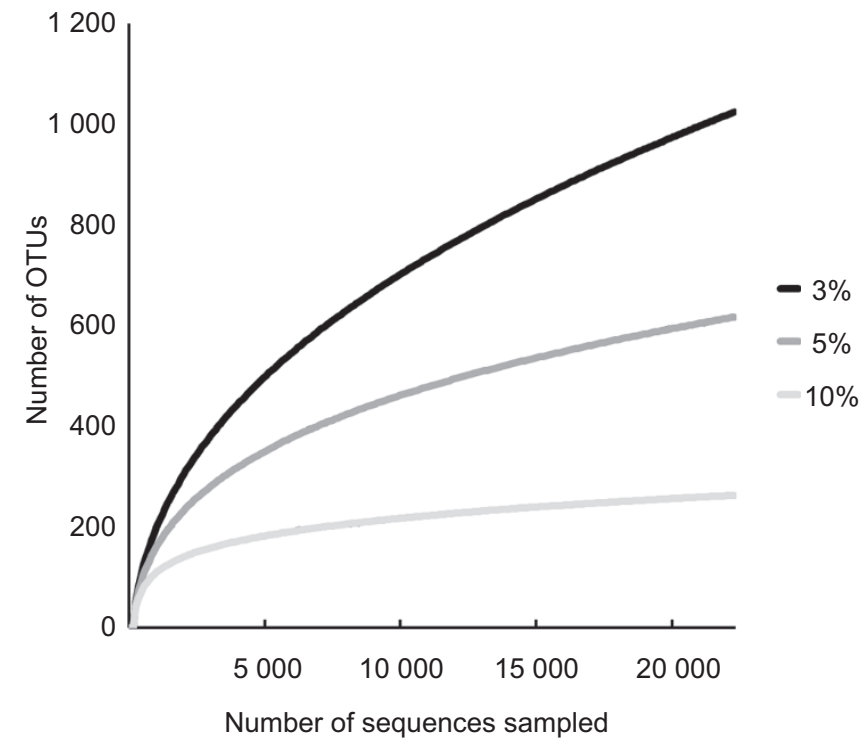

Figure 1 Rarefaction curves of control group (PT, prior to treatment). The relationship between OTUs found and number of sequences sampled was observed at $3 \%, 5 \%$ and $10 \%$ dissimilarity levels and the corresponding curves were plotted. OTU, operational taxonomic unit. 
sequences are sufficient or what sampling depth is needed depends on the goal of the study. As few as 100 sequences per sample were sufficient to detect the major patterns of variation among the microbial communities in the guts of diverse mammals. ${ }^{17}$ Depth of coverage of about 1000 sequences per sample seems to provide a good balance between number of samples and depth of sampling. ${ }^{18}$ The number of sequences analyzed in our study ( 2629 per sample) was well above that recommended and can be considered reasonable. Compared with the oral microbial communities determined by cultivation or traditional cloning and sequencing, these results from pyrosequencing analysis showed much higher diversity. However, if the goal is complete characterization of all phylotypes in a sample or group, additional sequences will be required to determine the detailed diversity of oral microflora, especially when many species are rare or the diversity is high.

\section{Composition of the bacterial community}

The current technology is generally much more effective in the identification of higher level taxonomic assignments such as phyla, orders and genera, than species or strains. ${ }^{19}$ After eliminating unclassified sequences (4 038 sequences, $2.7 \%$ ), 140 different genera were identified from pooled samples of seven time points. The top three genera in the control group (PT) included, in order of prevalence at the time point PT, Neisseria (16.19\% of the sequences taxonomically assigned at the genus level), Streptococcus (15.58\%) and Capnocytophaga (15.04\%). By contrast, the top three genera varied significantly across different time points during radiotherapy (Figure 2). Among all genera, 11 genera (Streptococcus, Actinomyces, Veillonella, Capnocytophaga, Derxia, Neisseria, Rothia, Prevotella, Granulicatella, Luteococcus and Gemella), which were found in all subjects, varied in relative abundance
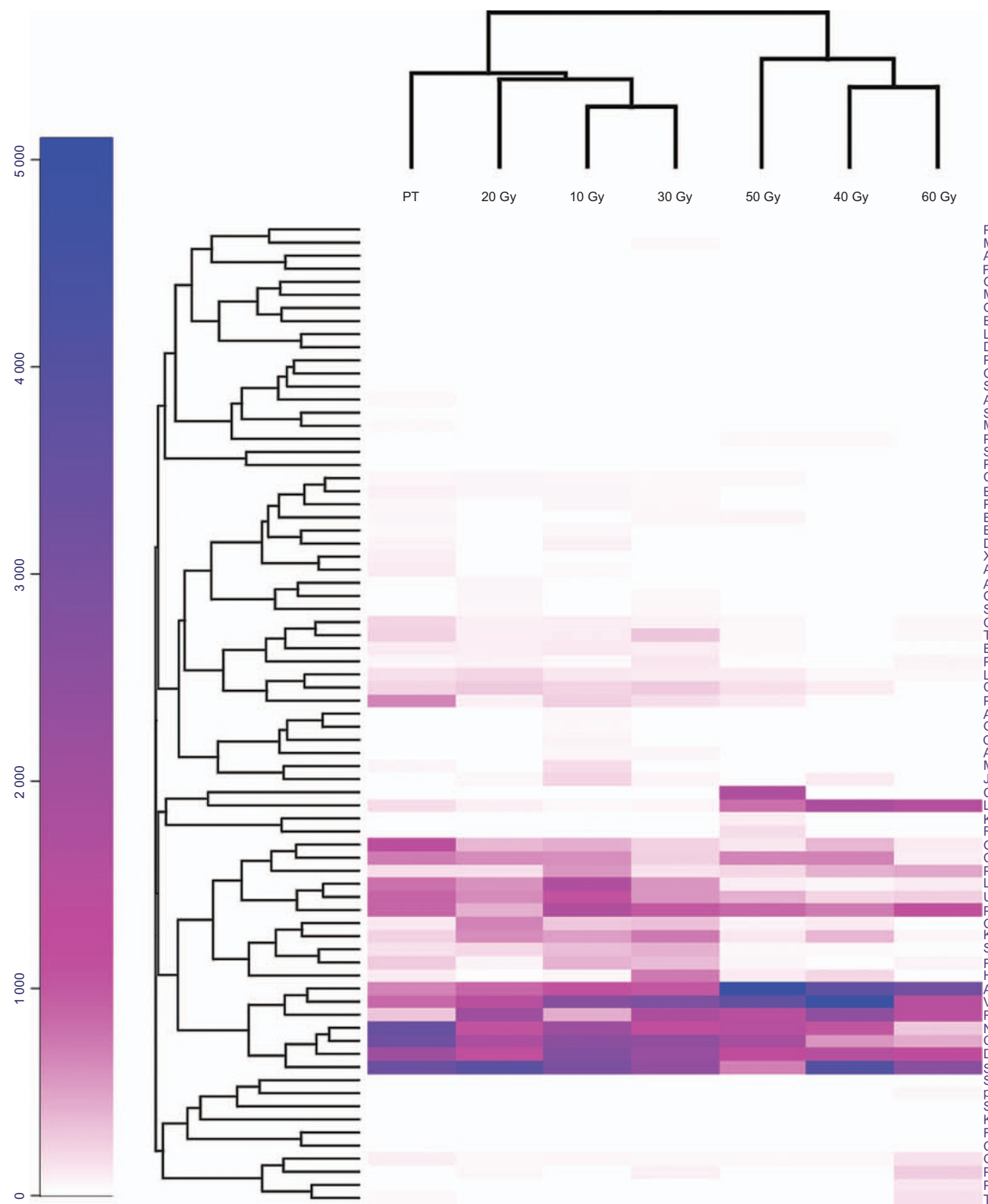

Paptococcus Megasphaera Filifactor Mogibacterium Odoribacter Branchiibius Lutaonella Desulfomicrobium Peptostreptococ Solobacterium Abiotrophia Spirochaeta Mycoplasma Pseudogulbenkiania Paptostreptococcaceae Cardiobacterium Bergayella Paludibacter Bergeriella Dialister
Xylanibacter Aggregatibacter Actinobaculum Ottowia Synergistaceae
Centipeda Treponema Eikenella Phocaeicola Luteococcus Porphyromonas Atopobium Oribacterium Olsenella Anaeroglobus
Moryella
Johnsonella Citrobacter Lactobacillus Kinneretia aoultella Granulicatella Pseudomonas eptotrichia nknow revotella Kingella Selenomonas usobacterium ctinomyces (allonerl Neisseria Derxia Streptococcus pynergistaceae Scardovia
Klebsiella Klebsiella Castidiosipila Gemella Pyramidobacter Pseudoramibacter Tannerella

Figure 2 Heat map analysis of the top 75 genera detected among seven time points. The color of each column represents the number of sequences of corresponding genus. The phylogenetic trees generated by the R program were used to estimate the distances based on genus composition. 


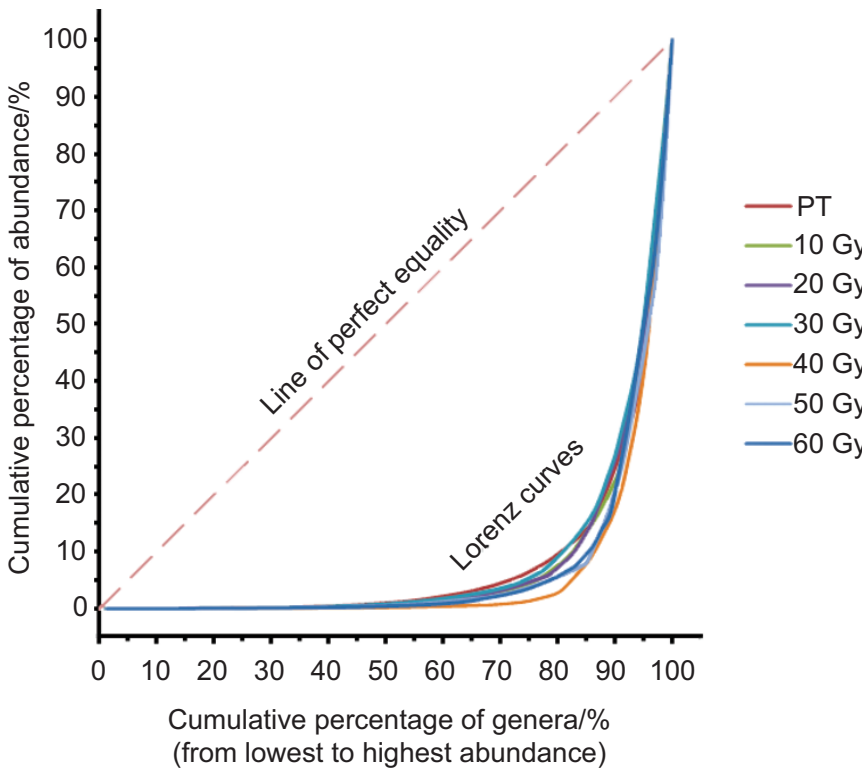

Figure 3 Lorenz curves representing communities from seven time points. The percentage of genera was plotted on the $X$-axis in ascending order of sequences they occupied (from lowest to highest abundance), the percentage of number of sequences on the $Y$-axis. The 'line of perfect equality' was depicted by a straight line in which ' $N$ "\% of the genera would always had ' $N$ \% of the sequences. The observed Lorenz curves of seven time points were far away from the line of perfect equality and formed an area shown in gray. The larger this area, the more uneven a community was.

during the course of radiotherapy (illustrated in detail in Figure 2). Although from our data, it is not possible to draw any conclusions regarding their pathogenicity, pathogenic taxa are suspected from previous studies. Streptococcus and other caries-related bacteria such as Veillonella and Actinomyces fluctuated significantly and accounted for a large proportion of the bacterial communities. These top three genera interact with each other in the oral cavity, and they may play an important role in the development of dental caries. ${ }^{20-22}$ It is known that Granulicatella is very fastidious and difficult to cultivate. In our study, this genus was dramatically reduced in dental plaque following radiation. It is therefore tempting to speculate that Granulicatella is unlikely to contribute to the pathogenesis of post-radiation diseases such as radiation caries. Derxia, which has also been found in tropical soils, ${ }^{23}$ and Luteococcus, which was also isolated from human blood and the peritoneum, ${ }^{24-25}$ fluctuated differently, but little information about its pathogenicity in oral cavity is available so far. Other predominant genera, such as Rothia, Prevotella, Capnocytophaga and Neisseria, might be involved in the susceptibility of an individual to periodontal disease. ${ }^{16}$

It was possible that the observed bacterial composition might be influenced by biases in the $16 \mathrm{~S}$ rRNA sequencing technology. There were multiple possible sources of bias including the method of DNA extraction, PCR amplification, target region selection, sequence screening, etc. For example, the genus Prevotella may be predominant when the hypervariable V1-V3 region is targeted. ${ }^{26}$ However, this region can provide results similar to Sanger sequencing and obtain representational characterization of microbial communities. In addition, most of the pyrosequencing contain none or only a few errors which are caused by homopolymers (repeated nucleotides). These errors may be interpreted as a rare OTU, inflating richness estimates. ${ }^{19}$ In consideration of the complexity of Next Generation Sequencing platform, more effort should be made to reduce the biases of current technology.
Among all genera, 50\% of all sequences were comprised of the top five genera including Streptococcus, Veillonella, Actinomyces, Capnocytophaga and Derxia. Moreover, the top 10 and top 30 genera constituted roughly $80 \%$ and $90 \%$ of the total sequences in relative abundance, respectively. Therefore, it was reasonable to speculate that the overall sequence distribution was unequal. When dealing with the issue of evenness, the Lorenz curve developed by Max O. Lorenz was originally used to assess inequality of social wealth distribution, and has been recently introduced to studies of biodiversity. ${ }^{27}$ In this research, the sequence distribution of communities before and during radiotherapy was represented by Lorenz curves (Figure 3). Ideally, a perfectly equal sequence distribution would be one in which every genus has the same number of sequences (line of perfect equality in Figure 3). However, the shapes of Lorenz curves in our study were far away from the perfect evenness line, indicating that the microbial communities were dominated by several major taxa and the genera evenness was low, even though the diversity was high.

\section{Cluster analysis}

The assessment of differences between microbial communities is critical for understanding large-scale trends in microbial ecology. By comparing the compositions of oral communities from different time periods, we can learn how specific factors affect community development and how species or individuals associate with each other. ${ }^{28}$ Cluster analysis using weighted UniFrac metrics (Figure 4) found that samples before radiotherapy (PT) and the early stages of radiotherapy (10 Gy, $20 \mathrm{~Gy}$ and $30 \mathrm{~Gy}$ ) formed a cluster distinct from the time points at later stages of radiotherapy (40 Gy, 50 Gy and $60 \mathrm{~Gy}$ ), indicating that there existed temporal variations in microbial communities through the course of treatment. Comparing the cluster analysis with the phylogenetic tree generated by the R program showed similar results for the distances based on genus composition (Figure 2).

In summary, the bacterial diversity and temporal variation of oral microbiota in patients receiving head-and-neck radiotherapy were investigated by pyrosequencing. The detailed richness and relative abundance of 140 genera were found and the temporal fluctuations of the microbial communities were also observed across seven time points before and during radiotherapy. Moreover, the low evenness of genera in relative abundance was revealed by Lorenz curves. The present study demonstrated that high-throughput pyrosequencing

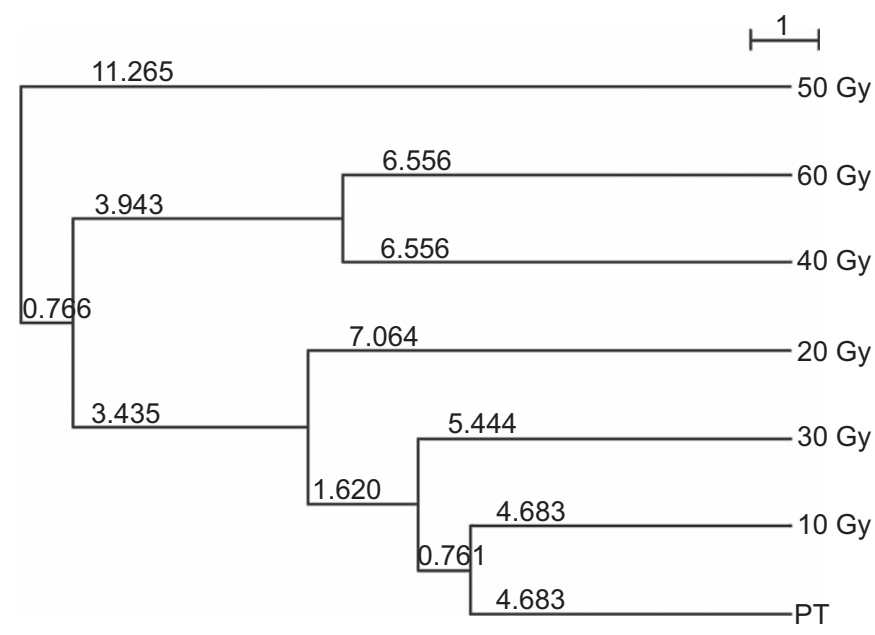

Figure 4 Differentiation in microbial communities before and during radiotherapy. The tree was measured using the weighted UniFrac algorithm. 
facilitated the evaluation of microbiome in the diseased condition. This technique provided valuable information about the profiles of oral microbial communities and potential pathogens during radiotherapy, and may help guide the clinical medication and microbial intervention during the course of treatment.

\section{ACKNOWLEDGEMENTS}

We express our sincere appreciation to all the patients who took part in this study. This work was supported by a grant from the National Natural Science Foundation (No. 81070826/30872886) of China and partly sponsored by Shanghai Rising-Star Program (No. 12QH1401400). Patient enrollment was funded by the Shanghai Jiao Tong University (Grant No. YG2011MS67).

1 Walker MP, Wichman B, Cheng AL et al. Impact of radiotherapy dose on dentition breakdown in head and neck cancer patients. Pract Radiat Oncol 2011; 1(3): 142148.

2 Shih A, Miaskowski C, Dodd MJ et al. Mechanisms for radiation-induced oral mucositis and the consequences. Cancer Nurs 2003; 26(3): 222-229.

3 Rothwell BR. Prevention and treatment of the orofacial complications of radiotherapy. J Am Dent Assoc 1987; 114(3): 316-322.

4 Whitmyer CC, Waskowski JC, Iffland HA. Radiotherapy and oral sequelae: preventive and management protocols. J Dent Hyg 1997; 71(1): 23-29.

5 Nederfors T. Xerostomia and hyposalivation. Adv Dent Res 2000; 14: 48-56.

6 MacFarlane TW, Mason DK. Changes in the oral flora in Sjögren's syndrome. J Clin Pathol 1974; 27: 416-419.

7 Leung WK, Jin LJ, Samaranayake LP et al. Subgingival microbiota of shallow periodontal pockets in individuals after head and neck irradiation. Oral Microbiol Immunol 1998; 13(1): 1-10.

8 Aas JA, Paster BJ, Stokes LN et al. Defining the normal bacterial flora of the oral cavity. J Clin Microbiol 2005; 43(11): 5721-5732.

9 He XS, Shi WY. Oral microbiology: past, present and future. Int J Oral Sci 2009; 1(2): 47-58.

$10 \mathrm{Li} \mathrm{Y,} \mathrm{Ku} \mathrm{CY,} \mathrm{Xu} \mathrm{J} \mathrm{et} \mathrm{al.} \mathrm{Survey} \mathrm{of} \mathrm{oral} \mathrm{microbial} \mathrm{diversity} \mathrm{using} \mathrm{PCR-based} \mathrm{denaturing}$ gradient gel electrophoresis. J Dent Res 2005; 84(6): 559-564.

11 Rasiah IA, Wong L, Anderson SA et al. Variation in bacterial DGGE patterns from human saliva: over time, between individuals and in corresponding dental plaque microcosms. Arch Oral Biol 2005; 50(9): 779-787.
12 Lazarevic V, Whiteson $\mathrm{K}$, Hernandez D et al. Study of inter- and intra-individual variations in the salivary microbiota. BMC Genomics 2010; 11: 523.

13 Carr AJ, Ng WF, Figueiredo F et al. Sjogren's syndrome-an update for dental practitioners. Br Dent J 2012; 213(7): 353-357.

14 Shao ZY, Tang ZS, Yan C et al. Effects of intensity-modulated radiotherapy on human oral microflora. J Radiat Res 2011; 52(6): 834-839.

15 Margulies M, Egholm M, Altman WE et al. Genome sequencing in open microfabricated high density picolitre reactors. Nature 2005; 437(7057): 376-380.

16 Ling Z, Kong J, Jia P et al. Analysis of oral microbiota in children with dental caries by PCR-DGGE and barcoded pyrosequencing. Microb Ecol 2010; 60(3): 677-690.

17 Ley RE, Hamady M, Lozupone $\mathrm{C}$ et al. Evolution of mammals and their gut microbes. Science 2008; 320(5883): 1647-1651.

18 Hamady M, Knight R. Microbial community profiling for human microbiome projects: tools, techniques, and challenges. Genome Res 2009; 19(7): 1141-1152.

19 Siqueira JJ, Fouad AF, Rocas IN. Pyrosequencing as a tool for better understanding of human microbiomes. J Oral Microbiol 2012; 4: 10.3402/jom.v4i0.10743. Epub 2012 Jan. 23.

20 Aas JA, Griffen AL, Dardis SR et al. Bacteria of dental caries in primary and permanent teeth in children and young adults. J Clin Microbiol 2008; 46(4): 1407-1417.

21 Huang Z, Meric G, Liu Z et al. luxS-based quorum-sensing signaling affects biofilm formation in Streptococcus mutans. J Mol Microbiol Biotechnol2009; 17(1): 12-19.

$22 \mathrm{He} \mathrm{Z}$, Wang Q, Hu Y et al. Use of the quorum sensing inhibitor furanone C-30 to interfere with biofilm formation by Streptococcus mutans and its luxS mutant strain. Int J Antimicrob Agents 2012; 40(1): 30-35.

$23 \mathrm{Xie} \mathrm{CH}$, Yokota A. Phylogenetic analyses of the nitrogen-fixing genus Derxia. J Gen Appl Microbiol 2004; 50(3): 129-135.

24 Collins MD, Lawson PA, Nikolaitchouk N et al. Luteococcus peritoneisp. nov., isolated from the human peritoneum. Int J Syst Evol Microbiol 2000; 50(Pt 1): 179-181.

25 Collins MD, Hutson RA, Nikolaitchouk $\mathrm{N}$ et al. Luteococcus sanguinis sp. nov., isolated from human blood. Int J Syst Evol Microbiol 2003; 53(Pt 6): 1889-1891.

26 Kumar PS, Brooker MR, Dowd SE et al. Target region selection is a critical determinant of community fingerprints generated by 16 S pyrosequencing. PLOS ONE 2011; 6(6): e20956.

27 Wittebolle L, Marzorati M, Clement L et al. Initial community evenness favours functionality under selective stress. Nature 2009; 458(7238): 623-626.

28 Chang Q, Luan Y, Sun F. Variance adjusted weighted UniFrac: a powerful beta diversity measure for comparing communities based on phylogeny. BMC Bioinformatics 2011; 12: 118.

(c) This work is licensed under a Creative Commons Attribution-NonCommercial-NoDerivative Works 3.0 Unported License. To view a copy of this license, visit http:// creativecommons.org/licenses/by-nc-nd/3.0 